University of Wollongong

Research Online

Australian Institute for Innovative Materials -

Papers

Australian Institute for Innovative Materials

2013

Single-pulse terahertz coherent control of spin resonance in the canted antiferromagnet YFeO3, mediated by dielectric anisotropy

Zuanming Jin

Shanghai University

Zoltan Mics

Max Planck Institute For Polymer Research

Guohong Ma

Shanghai University

Zhenxiang Cheng

University of Wollongong, cheng@uow.edu.au

Mischa Bonn

Max Planck Institute For Polymer Research

See next page for additional authors

Follow this and additional works at: https://ro.uow.edu.au/aiimpapers

Part of the Engineering Commons, and the Physical Sciences and Mathematics Commons

Research Online is the open access institutional repository for the University of Wollongong. For further information contact the UOW Library: research-pubs@uow.edu.au 


\title{
Single-pulse terahertz coherent control of spin resonance in the canted antiferromagnet $\mathrm{YFeO3}$, mediated by dielectric anisotropy
}

\begin{abstract}
We report on the coherent control of terahertz $(\mathrm{THz})$ spin waves in a canted antiferromagnet yttrium orthoferrite, YFeO3, associated with a quasiferromagnetic (quasi-FM) spin resonance at a frequency of $0.3 \mathrm{THz}$, using a single-incident $\mathrm{THz}$ pulse. The spin resonance is excited impulsively by the magnetic field component of the $\mathrm{THz}$ pulse. The intrinsic dielectric anisotropy of $\mathrm{YFeO} 3$ in the $\mathrm{THz}$ range allows for coherent control of both the amplitude and the phase of the excited spin wave. The coherent control is based on simultaneous generation of two interfering phase-shifted spin waves whose amplitudes and relative phase, dictated by the dielectric anisotropy of the YFeO3 crystal, can be controlled by varying the polarization of the incident $\mathrm{THz}$ pulse with respect to the crystal axes. The spatially anisotropic decay of the $\mathrm{THz}$-excited FM spin resonance in $\mathrm{YFeO}$, leading to an increasingly linear polarization of the $\mathrm{THz}$ oscillation at the spin resonance frequency, suggests a key role of magnon-phonon coupling in spin-wave energy dissipation. 2013 American Physical Society.
\end{abstract}

\section{Keywords}

terahertz, coherent, control, spin, resonance, canted, antiferromagnet, yfeo3, single, mediated, pulse, dielectric, anisotropy

Disciplines

Engineering | Physical Sciences and Mathematics

\section{Publication Details}

Jin, Z., Mics, Z., Ma, G., Cheng, Z., Bonn, M. \& Turchinovich, D. (2013). Single-pulse terahertz coherent control of spin resonance in the canted antiferromagnet YFeO3, mediated by dielectric anisotropy. Physical Review B - Condensed Matter and Materials Physics, 87 (9), 094422-1-094422-5.

\section{Authors}

Zuanming Jin, Zoltan Mics, Guohong Ma, Zhenxiang Cheng, Mischa Bonn, and Dmitry Turchinovich 


\title{
Single-pulse terahertz coherent control of spin resonance in the canted antiferromagnet $\mathrm{YFeO}_{3}$, mediated by dielectric anisotropy
}

\author{
Zuanming Jin, ${ }^{1,2}$ Zoltán Mics, ${ }^{1}$ Guohong Ma, ${ }^{2}$ Zhenxiang Cheng, ${ }^{3}$ Mischa Bonn, ${ }^{1}$ and Dmitry Turchinovich $, 4,{ }^{1,}$ \\ ${ }^{1}$ Max Planck Institute for Polymer Research, Ackermannweg 10, 55128 Mainz, Germany \\ ${ }^{2}$ Department of Physics, Shanghai University, 99 Shangda Road, Shanghai 200444, People's Republic of China \\ ${ }^{3}$ Institute for Superconductor and Electronic Materials, University of Wollongong, Squires Way, North Wollongong, NSW 2500, Australia \\ ${ }^{4}$ DTU Fotonik, Technical University of Denmark, Ørsteds Plads 343, DK-2800 Kgs. Lyngby, Denmark
}

(Received 8 January 2013; published 25 March 2013)

\begin{abstract}
We report on the coherent control of terahertz $(\mathrm{THz})$ spin waves in a canted antiferromagnet yttrium orthoferrite, $\mathrm{YFeO}_{3}$, associated with a quasiferromagnetic (quasi-FM) spin resonance at a frequency of $0.3 \mathrm{THz}$, using a single-incident $\mathrm{THz}$ pulse. The spin resonance is excited impulsively by the magnetic field component of the $\mathrm{THz}$ pulse. The intrinsic dielectric anisotropy of $\mathrm{YFeO}_{3}$ in the $\mathrm{THz}$ range allows for coherent control of both the amplitude and the phase of the excited spin wave. The coherent control is based on simultaneous generation of two interfering phase-shifted spin waves whose amplitudes and relative phase, dictated by the dielectric anisotropy of the $\mathrm{YFeO}_{3}$ crystal, can be controlled by varying the polarization of the incident $\mathrm{THz}$ pulse with respect to the crystal axes. The spatially anisotropic decay of the THz-excited FM spin resonance in $\mathrm{YFeO}_{3}$, leading to an increasingly linear polarization of the $\mathrm{THz}$ oscillation at the spin resonance frequency, suggests a key role of magnon-phonon coupling in spin-wave energy dissipation.
\end{abstract}

DOI: 10.1103/PhysRevB.87.094422

PACS number(s): 85.75.-d, 76.50.+g, 78.47.J-, 75.50.Ee

\section{INTRODUCTION}

Ultrafast control of spins in solids has been receiving increasing attention because of its potential applications in spintronics and spin-based information processing. ${ }^{1}$ The direct, magnetic field-induced, ultrafast excitation and control of spin waves is most advantageous for these purposes. However, due to the lack of efficient ultrafast sources of direct magnetic excitation, the ultrafast magnetization dynamics in magnetically ordered materials was until recently initiated and controlled indirectly, using various optically activated mechanisms. For example, the inverse Faraday effect based on Raman-type nonlinear optical process, ${ }^{2-4}$ dynamic momentum-space distribution of photoexcited spinpolarized carriers, ${ }^{5}$ optical Stark effect, ${ }^{6}$ thermal magnetic anisotropy change, ${ }^{7}$ and lattice distortion ${ }^{8}$ has been employed to excite the spin systems on femtosecond and picosecond timescales. In most of these cases, the spin excitation by ultrashort laser illumination of materials is accompanied by an undesirable thermal contribution, usually associated with electron dynamics that mediate the excitation of spin waves in the laser-induced process. Ideally, the ultrafast excitation and manipulation of spin dynamics in future spintronic systems should be heat-free, i.e., not leading to any thermal load on the device. In this paper, we directly use the magnetic field component of an ultrashort terahertz $(\mathrm{THz})$ pulse in a $\mathrm{THz}$ time-domain spectroscopy (THz-TDS) experiment to create and manipulate the spin wave on an ultrafast timescale.

\section{THz EXCITATION AND COHERENT CONTROL OF SPIN RESONANCES VIA ZEEMAN TORQUE}

THz-TDS has become an invaluable tool in experimental materials science studies. ${ }^{9-12}$ It is based on direct amplitudeand phase-resolved measurement of ultrashort and broadband, usually single-cycle, electromagnetic transients with the frequency spectrum covering the $\mathrm{THz}$ band, transmitted through the materials under study. The key advantage of this technique is that the subcycle temporal resolution in the detection of propagating $\mathrm{THz}$ transients allows direct observation of the dynamics of light-matter interaction. Traditionally, phenomena of dielectric origin, i.e., related to the coupling of the electric field component of the $\mathrm{THz}$ transient to electronic or ionic systems, have been studied, such as linear ${ }^{11,12}$ and nonlinear ${ }^{13-15}$ electron response in semiconductors and polar lattice vibration in crystals. ${ }^{16,17}$ Recently, the ultrafast $\mathrm{THz}$ spectroscopy also proved to be an effective experimental method of detection and manipulation of magnetization in materials utilizing the magnetic field component of the $\mathrm{THz}$ transient through magnetic dipole transitions. ${ }^{18-22}$ In this fashion, the ultrafast magnetization dynamics in ferromagnetic $(\mathrm{FM})^{23}$ and antiferromagnetic (AFM) materials ${ }^{24-27}$ have been investigated.

The mechanism of observation of magnetization dynamics in a THz-TDS experiment is based on the free induction decay (FID) of an impulsively excited spin precession, as shown in Fig. 1(a). The magnetic field component of the THz pulse $\mathbf{B}_{\mathrm{THz}}$, oriented orthogonally to the material macroscopic magnetization vector $\mathbf{M}$, induces a Zeeman torque $\mathbf{T} \propto$ $\mathbf{M} \times \mathbf{B}$ on the $\mathbf{M}$ vector. As a result of the impulsive Zeeman torque, the tilted macroscopic magnetization vector $\mathbf{M}$ starts precessing around its unperturbed orientation at the Larmor frequency, leading to circularly polarized electromagnetic emission $\mathbf{E}_{\mathrm{THz}} \propto \partial^{2} \mathbf{M} / \partial t^{2}$ (where $t$ is time) from the rotating magnetic dipole, in the direction of an unperturbed $\mathbf{M}$ vector, at the Larmor frequency. ${ }^{24-27}$

The excitation of spin waves by an impulsive, (sub-) picosecond, magnetic field transient can be considered quasiinstantaneous. However, the natural decay of the spin waves, which can be assigned to magnon-magnon or magnon-phonon interactions, ${ }^{25,28}$ usually takes place on the timescale of tens to hundreds of picoseconds. ${ }^{29,30}$ Potential applications in ultrafast spin-based information processing and spintronics require 

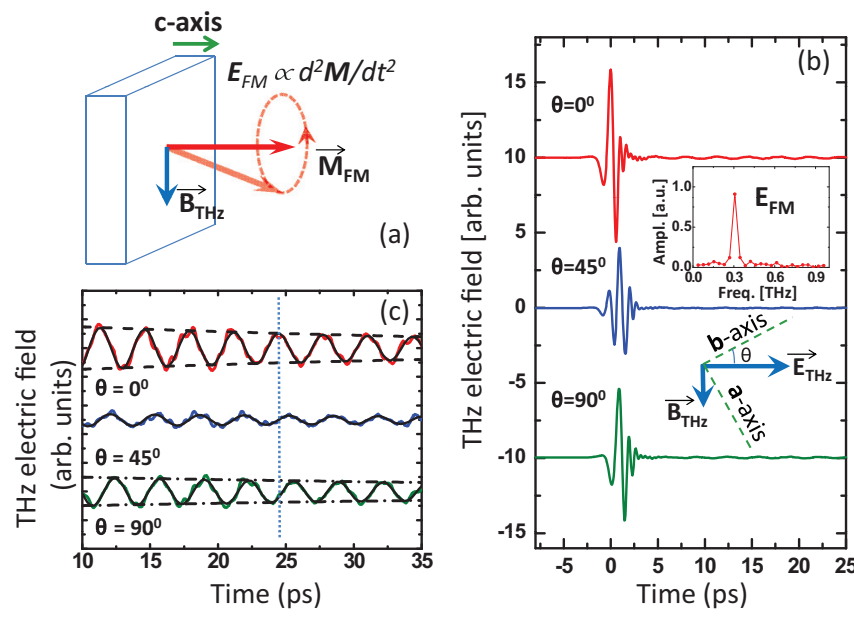

FIG. 1. (Color online) (a) Mechanism of $\mathrm{THz}$ emission by spin resonance in a $c$-cut $\mathrm{YFeO}_{3}$ crystal. (b) $\mathrm{THz}$ waveform transmitted through crystal at selected azimuthal angles of $\theta=0^{\circ}, 45^{\circ}$, and $90^{\circ}$, vertically offset for clarity. Orientation of the $\mathrm{THz}$ pulse polarization with respect to the crystal axes of $\mathrm{YFeO}_{3}$ crystal is shown in the lower inset of (b). (c) A zoom of the oscillating part of the THz electric field in the time window of 10-35 ps, together with the curve-fitting results (solid lines). Dashed lines are single-exponential decay fits. The vertical dotted line emphasizes the phase difference between the different $\mathrm{THz}$ traces. The Fourier amplitude of the oscillatory component $\left(E_{\mathrm{FM}}\right)$ at $\theta=0^{\circ}$ is shown in the upper inset of (b).

active manipulation of the amplitude or the phase, or both, of transient magnetization on a much faster picosecond timescale.

Such active ultrafast manipulation over an otherwise long-lasting spin excitation can be achieved using coherent control. ${ }^{31}$ This technique usually utilizes a pair of controllably delayed excitation pulses. The first pulse (pump) excites a long-lasting spin wave, while the time-delayed second pulse (control) excites yet another, phase-delayed replica of this spin wave, making it interfere with the first one. The relative phase between these two spin waves is governed by the time delay between the pump and the control pulses: if this time delay equals an even number of half-periods of spin precession, the constructive interference between these two partial waves leads to enhancement of the spin excitation by the action of the control pulse; whereas if this time delay equals an odd number of half-periods, destructive interference quenches spin excitation. Recently, Kampfrath et al. reported the coherent control of spin waves by using a pair of intense copolarized $\mathrm{THz}$ pulses for pump and control, and by using a time-delayed optical probe pulse for the observation of the spin waves in the antiferromagnet $\mathrm{NiO} .{ }^{25}$ Similarly, an all- $\mathrm{THz}$ experiment involving a pair of much weaker $\mathrm{THz}$ pulses was used for coherent control of the spin precession in yttrium orthoferrite $\left(\mathrm{YFeO}_{3}\right){ }^{27}$

In this paper, we show the coherent control of the quasi-FM spin resonance at the frequency of $0.3 \mathrm{THz}$ in the canted AFM crystal $\mathrm{YFeO}_{3}$ using only a single incident $\mathrm{THz}$ pulse. The amplitude and the phase of the $\mathrm{THz}$ spin waves can be controlled coherently by choosing the polarization of the incident $\mathrm{THz}$ pulse with respect to the symmetry axes of the anisotropic crystal. The dielectric anisotropy of the crystal leads to the decomposition of an incident $\mathrm{THz}$ field into phase-shifted ordinary and extraordinary rays. We demonstrate that the polarization-dependent interference of these rays can be used to control both the amplitude and the phase of the resulting spin oscillation. The efficient materials for ultrafast spintronics with spin resonances in (sub-) THz range, such as rare-earth orthoferrites, are strongly anisotropic. Through our approach, we add polarization as a novel degree of freedom for coherent manipulation of ultrafast magnetization dynamics.

\section{SAMPLE AND EXPERIMENT}

Our $\mathrm{YFeO}_{3}$ crystal was grown by the floating zone method, was polished on both sides, and had a thickness of $d=$ $1.33 \mathrm{~mm}$. All our measurements were performed at room temperature, i.e., well below the Néel temperature of $T_{N}=$ $645 \mathrm{~K}$. The magnetic properties of $\mathrm{YFeO}_{3}$ with orthorhombic structure depend on the crystal orientation and are governed by the magnetization of the $\mathrm{Fe}^{3+}$ sublattice. The electron spins associated with the nearest-neighbor $\mathrm{Fe}^{3+}$ ions along the $c$ axis and $a$ axis are aligned, respectively, in a parallel and an antiparallel fashion. This results in the existence of quasi-FM and quasi-AFM spin modes in the crystal. ${ }^{32}$ The resonant frequencies of FM and AFM spin modes in $\mathrm{YFeO}_{3}$ are 0.30 and $0.53 \mathrm{THz}$, respectively. ${ }^{33}$ In this paper, we study the dynamics of the FM spin mode in $\mathrm{YFeO}_{3}$ designated as $\mathbf{M}_{\mathrm{FM}}$ in Fig. 1(a). The AFM magnetization vector is not plotted in the schematic and is not important in this paper for reasons related to the excitation geometry, as explained later.

In our experiments, the $\mathrm{YFeO}_{3}$ crystal was $c$ cut, and the propagation direction of incident $\mathrm{THz}$ radiation was coaligned with the $c$ axis, i.e., along the magnetization vector $\mathbf{M}_{\mathrm{FM}}$ of the crystal. This geometry has two important consequences. First, the magnetic field component of the $\mathrm{THz}$ pulse, whose magnetic field vector $\mathbf{B}_{\mathrm{THz}}$ belongs to the plane defined by the $a$ and $b$ axes, is always orthogonal to $\mathbf{M}_{\mathrm{FM}}$. Therefore, independent of its polarization with respect to the $a$ and $b$ axes, the $\mathrm{THz}$ pulse incident along the $c$ axis always has the strongest interaction with the $c$ axis-oriented $\mathbf{M}_{\mathrm{FM}}$ via maximum Zeeman torque $\mathbf{T} \propto \mathbf{M}_{\mathrm{FM}} \times \mathbf{B}_{\mathrm{THz}}$ [see Fig. 1(a) and the preceding discussion of the $\mathrm{THz}$ spin-wave excitation principle]. Second, in this geometry, the $\mathrm{THz}$ excitation of precession of the $a$ axis-oriented AFM magnetization vector does not enable FID emission along the $c$ axis (i.e., in the direction of propagation of the $\mathrm{THz}$ pulse), thus excluding the presence of the AFM effect in our measurement.

The $\mathrm{YFeO}_{3}$ crystal, like other rare-earth orthoferrites, ${ }^{33}$ has a sizeable dielectric anisotropy at THz frequencies along the $a$ and $b$ axes, producing both birefringence and dichroism. ${ }^{34,35}$ This anisotropy might be attributed to the eigenvectors of unstable $\Gamma$-point phonon modes, the $\Gamma_{15}$ and $\Gamma_{25}$ modes, which involve off-centering of the $\mathrm{Y}$ cation and oxygen displacements, respectively. ${ }^{36}$ This phonon-originated dielectric anisotropy of $\mathrm{YFeO}_{3}$, crucially important for the single-pulse coherent control effect, is characterized in detail later.

Our experiments were performed in a standard linear transmission-mode THz-TDS configuration. The single-cycle $\mathrm{THz}$ pulses were generated using optical rectification of 800-nm, 100-fs Ti:sapphire laser pulses, and detected by free-space electro-optic sampling, in $\langle 110\rangle$-oriented ZnTe crystals. ${ }^{9,10}$ The $\mathrm{THz}$ pulse spectrum covered the 
range 0.1-2.7 $\mathrm{THz}$, typical for this kind of experimental arrangement. The $\mathrm{THz}$ electric field strength did not exceed the value of $\sim 1 \mathrm{kV} / \mathrm{cm}$; therefore, the peak magnetic field was under $0.3 \mathrm{mT}$. The $\mathrm{THz}$ beam path in the spectrometer was purged with dry nitrogen in order to minimize absorption of $\mathrm{THz}$ radiation by atmospheric water vapor. The electric field of the incident $\mathrm{THz}$ pulse was linearly polarized in a horizontal plane. In addition, a THz polarizer with a horizontally oriented transmission axis was placed after the sample, ensuring that only this component of the $\mathrm{THz}$ field was detected. In the following, by the $\mathrm{THz}$ polarization orientation, we always refer to the orientation of the electric field vector of the THz signal.

Figure 1(b) shows the THz waveforms transmitted through the $\mathrm{YFeO}_{3}$ crystal oriented at different rotational (azimuthal) angles $\theta$ between the polarization of an incident $\mathrm{THz}$ pulse and the $b$ axis of the crystal, which is shown in the lower inset of Fig. 1(b). The azimuthal angles $\theta=0^{\circ}$ and $\theta=90^{\circ}$ correspond to the THz polarization along the $b$ and $a$ axis of the crystal, respectively. The measured $\mathrm{THz}$ waveforms consist of two components: the main part of the broadband $\mathrm{THz}$ pulse centered $\sim 0$ ps time delay and a slowly decaying oscillation caused by the FID emission of the FM spin resonance at a frequency of $0.30 \mathrm{THz}$. The Fourier transform of this oscillation ( $\left.E_{\mathrm{FM}}\right)$ at $\theta=0^{\circ}$ is shown in the inset of Fig. 1(b). The $\mathrm{YFeO}_{3}$ crystal birefringence causes a time delay between the THz pulses polarized along the $b$ and $a$ axes of the crystal (i.e., $\theta=0^{\circ}$ and $90^{\circ}$ ). When the $\mathrm{THz}$ polarization is oriented at $\theta=45^{\circ}$, the $\mathrm{THz}$ pulse experiences maximum anisotropy; as a result, its main part is composed of two delayed parts.

\section{DATA ANALYSIS AND DISCUSSION}

The implications of changing the angle $\theta$ between the crystal axes and the polarization of an incident $\mathrm{THz}$ pulse on the spin-wave FID THz emission are emphasized in Fig. 1(c). The solid lines are exponentially decaying sine-wave fits to the experimental data. The dashed lines show the exponential decay of the amplitude of the oscillation. The experimental data, apart from the FM spin resonance at $0.3 \mathrm{THz}$, features signature of residual water vapor absorption in the $\mathrm{THz}$ spectrometer beam path, adding a small modulation to the FM-mode signal. From the THz traces shown in Fig. 1(c), it is apparent that both the amplitude and the phase of the THz FM resonance signal strongly depend on the rotational angle $\theta$. The amplitude of the FID signal from $\mathrm{THz}$ spin waves can be changed by about a factor of two, and its phase can be shifted by a little over $\pi / 2$.

These observations cannot be fully explained by the simple mechanism of $\mathrm{THz}$ spin-wave excitation described earlier. From the geometry of spin-wave excitation shown in Fig. 1(a), we would expect polarization of the THz pulse in the plane of $a$ and $b$ axes to produce a maximum Zeeman torque, resulting in an equally strong FM spin mode precession, which is clearly not observed. In order to understand the dependence of the amplitude and the phase of the spin waves on the azimuthal angle $\theta$, we need to consider the complex dielectric function of the $\mathrm{YFeO}_{3}$ crystal. Fig. 2(a) and 2(b) shows the refractive index and the absorption spectra of our $c$-cut crystal measured along $a$ and $b$ axes, respectively. In retrieving the optical constants, we assumed the magnetic susceptibility $\mu \approx 1$, justified by

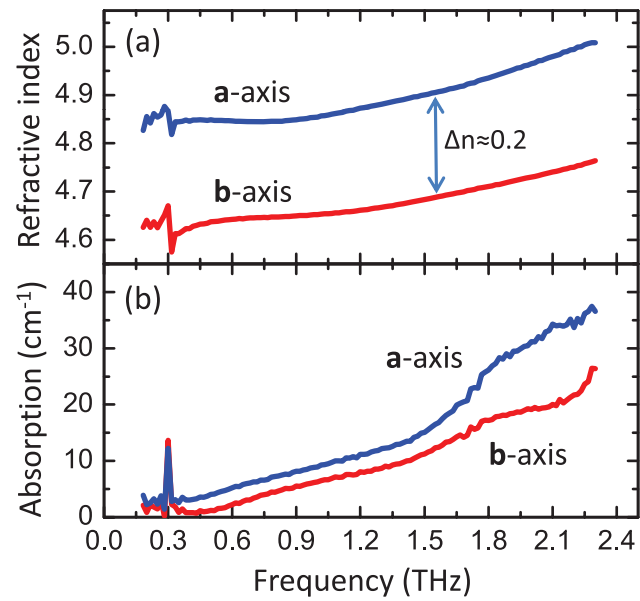

FIG. 2. (Color online) (a) Refractive indices $\mathrm{n}(\omega)$ and (b) absorption coefficients $\alpha(\omega)$ of a $c$-cut $\mathrm{YFeO}_{3}$ crystal measured with THz polarization along $a$ (blue curves) and $b$ axes (red curves), respectively.

a much smaller contribution of magnetic susceptibility to the complex refractive index compared to the dielectric one, even at the magnetic resonance frequency in orthoferrites. ${ }^{37}$

The refractive index and the absorption spectra shown in Fig. 2 have two contributions: an isolated FM resonance feature at a frequency of $0.3 \mathrm{THz}^{26,27}$ and the dispersive feature covering the entire frequency window of an experimentthe low-frequency tail of the resonances corresponding to unstable $\Gamma$-point phonon modes in the crystal at frequencies $\sim 10 \mathrm{THz}^{36}$ These phonon resonances cause the strong THzrange dielectric anisotropy of $\mathrm{YFeO}_{3}$. The birefringence of our sample along $a$ - and $b$-crystal axes $\Delta n=n_{a}-n_{b}$ is found to be $\sim 0.2$ over the whole spectral range of our experiment.

Let us consider the interaction of a linearly polarized $\mathrm{THz}$ pulse with FM spin resonance in such an anisotropic material. The electric field of the incident $\mathrm{THz}$ pulse $\mathbf{E}_{\mathrm{THz}}^{\mathrm{in}}$ enters the crystal at an arbitrary polarization angle $\theta$ [inset of Fig. 1(b)]. Propagating through the anisotropic crystal, the output $\mathrm{THz}$ pulse $\mathbf{E}_{\mathrm{THz}}^{\mathrm{out}}$ is represented by a sum of two orthogonal components coupled to $a\left(\mathbf{E}_{a}\right)$ and $b$ axes $\left(\mathbf{E}_{b}\right)$ of the crystal:

$$
\begin{aligned}
\mathbf{E}_{\mathrm{THz}}^{\text {out }} & =\mathbf{E}_{a}+\mathbf{E}_{b} \\
\mathbf{E}_{a} & =\mathbf{E}_{\mathrm{THz}}^{\mathrm{in}} \sin (\theta) \mathrm{e}^{\left(-\frac{\alpha_{a} d}{2}\right)} \mathrm{e}^{\left(i \frac{\omega n_{a} d}{c}\right)} \\
\mathbf{E}_{b} & =\mathbf{E}_{\mathrm{THz}}^{\mathrm{in}} \cos (\theta) \mathrm{e}^{\left(-\frac{\alpha_{b} d}{2}\right)} \mathrm{e}^{\left(i \frac{\omega n_{b} d}{c}\right)}
\end{aligned}
$$

where $\alpha_{a, b}$ and $n_{a, b}$ are the frequency-dependent absorption coefficients and refractive indices in the plane of $a$ and $b$ axes, respectively; $d$ is the crystal thickness; and $c$ is the speed of light in vacuum. These two orthogonally polarized broadband $\mathrm{THz}$ signals excite two spin waves at the frequency of FM resonance via their magnetic field components $\mathbf{B}_{a, b}=\mathbf{E}_{a, b} / c$. According to Eq. (1), these two spin waves have (1) different amplitudes according to the electric field projections on $a$ and $b$ axes defined by the azimuthal angle $\theta$ and the attenuation in the crystal defined by anisotropic absorption coefficient $\alpha_{a, b}$ and (2) different phases according to the anisotropic refractive index $n_{a, b}$. The phase difference, accumulated during the propagation of these two spin waves through the entire crystal, 
combined with different amplitudes of these two partial waves, results in their interference, leading to either enhancement or quenching of the resulting oscillation at the spin resonance frequency, i.e., to a coherent control effect. The preceding mechanism of $\mathrm{THz}$ spin-wave generation is most efficient if the driving $\mathrm{THz}$ field and the generated spin wave are perfectly phase matched. We estimate the refractive index mismatch between the driving $\mathrm{THz}$ field and the generated spin wave to be $\Delta n_{\text {mism }}=0.02-0.06$, depending on the crystal orientation. This estimate is based on the difference of measured refractive indices precisely at the spin resonance frequency of $0.30 \mathrm{THz}$, and $\sim 50-100 \mathrm{GHz}$ away from the resonance (Fig. 2). Based on such an index mismatch, we calculate the coherence length at the spin resonance frequency (the propagation distance at which the spin wave and the driving $\mathrm{THz}$ field acquire a relative phase of $\pi$ ) to be $l_{\text {cohr }}=8-25 \mathrm{~mm}$, which is much longer than the thickness of our crystal $d=1.33 \mathrm{~mm}$. Therefore, the assumption of perfect phase matching between the spin wave and the driving $\mathrm{THz}$ field can be well justified.

As mentioned earlier, in our experiment we detected the $\mathrm{THz}$ signal transmitted through the sample in the same (horizontal) polarization as the excitation $\mathrm{THz}$ pulse. By using the Jones matrix formalism, the horizontal and vertical components of the $\mathrm{THz}$ electric field at the position of the THz detector are given by $\mathbf{E}^{\text {horiz }}=\mathbf{E}_{b} \cos (\theta)+\mathbf{E}_{a} \sin (\theta)$ and $\mathbf{E}^{\text {vert }}=\mathbf{E}_{b} \sin (\theta)-\mathbf{E}_{a} \cos (\theta)$, respectively. As a result, the angular dependence of the amplitude of the $\mathrm{THz}$ field detected in the horizontal axis is

$$
\left|\mathbf{E}^{\text {horiz }}\right|=\left|\mathbf{E}_{\mathrm{THz}}^{\text {in }}\right| e^{\left(-\frac{1}{2} \alpha_{b} d\right)}\left[\cos ^{2}(\theta) \mathrm{e}^{i \Delta \Gamma} \mathrm{e}^{-\Delta \alpha d}+\sin ^{2}(\theta)\right]
$$

where $\Delta \alpha=\alpha_{a}-\alpha_{b}$ and $\Delta \Gamma=\Gamma_{a}-\Gamma_{b}=\frac{\omega d}{c} \Delta n$ are, respectively, the absorption coefficient difference and the phase retardation between two orthogonal polarization components of $\mathrm{THz}$ pulses, and hence between the excited spin waves. Eq. (2) therefore is our coherent control function, with a rotational angle $\theta$ between the incident $\mathrm{THz}$ polarization and the $b$ axis of the crystal as a control parameter.

In Fig. 3(a) and 3(b), the symbols show the dependency of the measured amplitude $\left(E_{\mathrm{FM}}\right)$ and phase of the FID signal of the FM-mode spin wave on the azimuthal angle $\theta$. The measured value of the signal phase shift shown in Fig. 3(b) matches the predicted maximum value $\Delta \Gamma=-1.47 \pi$ very well. The dependence of the spin-wave amplitude on the rotational angle $\theta$ shows a modulation of approximately a factor of 2 . The solid line in Fig. 3(a) represents the normalized coherent control function Eq. (2). Excellent agreement between the model and the measured data is observed. In the same figure, we show the separate contributions from the refractive index and the absorption coefficient anisotropies to the coherent control function Eq. (2). As dashed and dotted lines, we show this function including only refractive index anisotropy (assuming $\alpha_{a}=\alpha_{b}=0$ ) and only absorption anisotropy (assuming $n_{a}=$ $n_{b}$ ) of the $\mathrm{YFeO}_{3}$ crystal, respectively. We can see that both index and absorption contributions play a significant role in the observed effect.

As discussed earlier, the FM-mode spin resonance electromagnetic $\mathrm{THz}$ signal is induced by a rotating magnetic dipole and is thus inherently circularly polarized. Surprisingly, from the amplitude decay fits shown by the dashed lines in Fig. 1(c),

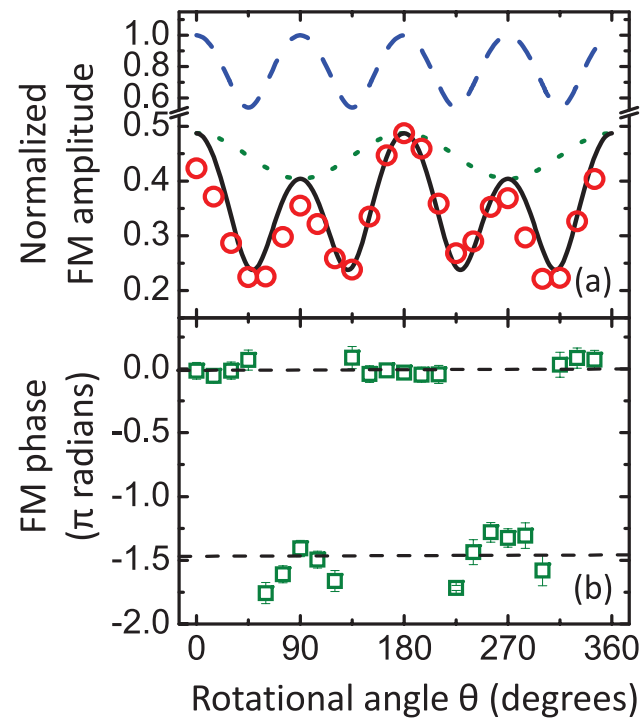

FIG. 3. (Color online) (a) The amplitude of the Fourier transform ( $E_{\mathrm{FM}}$; circular symbols) and (b) the phase (square symbols) of the FM spin mode emission as a function of rotational angle $\theta$. The normalized coherent control function Eq. (2), including full dielectric anisotropy at the FM spin resonant frequency (solid line), only index anisotropy (dashed line), and only absorption anisotropy (dotted line), is also shown in (a).

we find that the decay dynamics of two orthogonal linearly polarized components of this circularly polarized $\mathrm{THz}$ signal along the $b$ and $a$ axes (i.e., $\theta=0^{\circ}$ and $90^{\circ}$, respectively) have different exponential decay time constants: $\tau_{b}=40.2 \pm$ $2.2 \mathrm{ps}$ and $\tau_{a}=53.5 \pm 4.0 \mathrm{ps}$, respectively. The slower decay of the component polarized along the $a$ axis means that the initially circularly polarized FID signal becomes increasingly elliptical over time, with the long axis of the ellipse oriented along the $a$ axis of the crystal. ${ }^{26}$ Extrapolating this trend to long time delays, a nearly linearly polarized $\mathrm{THz}$ wave, i.e., a wave radiated by a linearly oscillating dipole (e.g., optical phonon), survives. However, this linearly polarized signal still oscillates at the Larmor frequency, i.e., at the frequency of the precessing magnetic dipole. This might be explained by the spin excitation decaying by (at least partly) transferring its energy to the lattice, as the FM spin resonance spectrally overlaps with the low-frequency tail of the phonon mode. The electric field component of the FM-mode THz electromagnetic emission can couple to the polar lattice of the crystal, leading to its periodic distortion. Hence, the crystal lattice may be caused to maintain the vibration at the frequency of the spin resonance, and the lattice anisotropy could cause the polarization orientation of a persisting lattice vibration.

\section{CONCLUSIONS}

We have demonstrated the coherent control of a quasiFM resonance at $0.30 \mathrm{THz}$ in a canted antiferromagnet $\mathrm{YFeO}_{3}$ using only a single $\mathrm{THz}$ pulse. The coherent control effect is mediated by the dielectric anisotropy of the $\mathrm{YFeO}_{3}$ crystal. Taking into account both the refractive index and the absorption coefficient anisotropy of the crystal, a coherent 
control function is developed, using the $\mathrm{THz}$ pulse polarization with respect to the crystal axes as a control parameter. In our 1.33-mm-thick $\mathrm{YFeO}_{3}$ crystal, the $\mathrm{THz}$ spin-wave amplitude could be modulated by a factor of 2 and the phase could be shifted by about $-3 \pi / 2$ simply by choosing the polarization of an incident $\mathrm{THz}$ pulse with respect to the crystal axes. A thicker crystal will allow for a stronger coherent control effect, including complete quenching of the spin wave. As mentioned previously, most efficient $\mathrm{THz}$ spintronic materials, such as rare-earth orthoferrites, are strongly anisotropic. ${ }^{33,35}$ The suggested method can thus further expand the range of capabilities in ultrafast $\mathrm{THz}$ manipulation of magnetization by adding one more degree of freedom, polarization, to existing coherent control schemes. Finally, significant spatial anisotropy in decay dynamics of $\mathrm{THz}$-excited spin wave was observed, leading to transition from a circularly polarized $\mathrm{THz}$ emission driven by the spin precession to a nearly linearly polarized emission, possibly driven by the persistent lattice vibration, at the Larmor frequency. The latter may directly manifest the transfer of spin-wave energy onto the lattice and is a subject of further investigation.

\section{ACKNOWLEDGMENTS}

We are grateful to Jørn M. Hvam, Alexey Scherbakov, and Matthias C. Hoffmann for useful discussions. We acknowledge partial financial support from the International Max Planck Research School, Max Planck Society, European Union Marie Curie Program (Career Integration Grant 334324 "LIGHTER"), Danish Council for Independent ResearchTechnology and Production Sciences (FTP Project ALFIE), Danish Proof-of-Concept Foundation (Grant No. 7.7 "Ultra high speed wireless data communications"), National Natural Science Foundation of China (Grant No. 11174195), and Australia Research Council (Future Fellowship). *turchino@mpip-mainz.mpg.de

${ }^{1}$ K. C. Nowack, F. H. L. Koppens, Yu. V. Nazarov, and L. M. K. Vandersypen, Science 318, 1430 (2007).

${ }^{2}$ A. V. Kimel, A. Kirilyuk, P. A. Usachev, R. V. Pisarev, A. M. Balbashov, and Th. Rasing, Nature 435, 655 (2005).

${ }^{3}$ A. V. Kimel, A. Kirilyuk, and T. Rasing, Laser Photon. Rev. 1, 275 (2007).

${ }^{4}$ Z. M. Jin, H. Ma, L. H. Wang, G. H. Ma, F. Y. Guo, and J. Z. Chen, Appl. Phys. Lett. 96, 201108 (2010).

${ }^{5}$ H. Ma, Z. Jin, G. Ma, W. Liu, and S. Tang, Appl. Phys. Lett. 94, 241112 (2009).

${ }^{6}$ J. A. Gupta, R. Knobel, N. Samarth, and D. D. Awschalom, Science 292, 2458 (2001).

${ }^{7}$ E. Beaurepaire, J.-C. Merle, A. Daunois, and J.-Y. Bigot, Phys. Rev. Lett. 76, 4250 (1996).

${ }^{8}$ M. Först, R. I. Tobey, S. Wall, H. Bromberger, V. Khanna, A. L. Cavalieri, Y.-D. Chuang, W. S. Lee, R. Moore, W. F. Schlotter, J. J. Turner, O. Krupin, M. Trigo, H. Zheng, J. F. Mitchell, S. S. Dhesi, J. P. Hill, and A. Cavalleri, Phys. Rev. B 84, 241104(R) (2011).

${ }^{9}$ B. Ferguson and X. Zhang, Nat. Mater. 1, 26 (2002).

${ }^{10}$ P. U. Jepsen, D. G. Cooke, and M. Koch, Laser Photon. Rev. 5, 124 (2011).

${ }^{11}$ J. Lloyd-Hughes and T.-I. Jeon, J. Infrared Milli. Terahz. Waves 33, 871 (2012).

${ }^{12}$ R. Ulbricht, E. Hendry, J. Shan, T. F. Heinz, and M. Bonn, Rev. Mod. Phys. 83, 543 (2011).

${ }^{13}$ M. C. Hoffmann and D. Turchinovich, Appl. Phys. Lett. 96, 151110 (2010).

${ }^{14}$ M. C. Hoffmann, B. S. Monozon, D. Livshits, E. U. Rafailov, and D. Turchinovich, Appl. Phys. Lett. 97, 231108 (2010).

${ }^{15}$ D. Turchinovich, J. M. Hvam, and M. C. Hoffmann, Phys. Rev. B 85, 201304(R) (2012).

${ }^{16}$ M. Schall, M. Walther, and P. U. Jepsen, Phys. Rev. B 64, 094301 (2001).

${ }^{17}$ M. Jewariya, M. Nagai, and K. Tanaka, Phys. Rev. Lett. 105, 203003 (2010).

${ }^{18}$ S. G. Chou, P. E. Stutzman, S. Wang, E. J. Garboczi, W. F. Egelhoff, and D. F. Plusquellic, J. Phys. Chem. C 116, 16161 (2012).
${ }^{19}$ J. L. M. van Mechelen, D. van der Marel, I. Crassee, and T. Kolodiazhnyi, Phys. Rev. Lett. 106, 217601 (2011).

${ }^{20}$ X. Wang, D. J. Hilton, L. Ren, D. M. Mittleman, J. Kono, and J. L. Reno, Opt. Lett. 32, 1845 (2007).

${ }^{21}$ A. Namai, M. Yoshikiyo, K. Yamada, S. Sakurai, T. Goto, T. Yoshida, T. Miyazaki, M. Nakajima, T. Suemoto, H. Tokoro, and S. Ohkoshi, Nat. Comm. 3, 1035 (2012).

${ }^{22}$ M. Nakajima, A. Namai, S. Ohkoshi, and T. Suemoto, Opt. Express 18, 18260 (2010).

${ }^{23}$ E. Beaurepaire, G. M. Turner, S. M. Harrel, M. C. Beard, J.-Y. Bigot, and C. A. Schmuttenmaer, Appl. Phys. Lett. 84, 3465 (2004).

${ }^{24}$ J. Nishitani, K. Kozuki, T. Nagashima, and M. Hangyo, Appl. Phys. Lett. 96, 221906 (2010).

${ }^{25}$ T. Kampfrath, A. Sell, G. Klatt, A. Pashkin, S. Mahrlein, T. Dekorsy, M. Wolf, M. Fiebig, A. Leitenstorfer, and R. Huber, Nat. Photon. 5, 31 (2011).

${ }^{26}$ R. Zhou, Z. Jin, G. Li, G. Ma, Z. Cheng, and X. Wang, Appl. Phys. Lett. 100, 061102 (2012).

${ }^{27}$ K. Yamaguchi, M. Nakajima, and T. Suemoto, Phys. Rev. Lett. 105, 237201 (2010).

${ }^{28}$ J. Stöhr and H. C. Siegmann, Magnetism: From Fundamentals to Nanoscale Dynamics (Springer-Verlag, Berlin, Heidelberg, 2006).

${ }^{29}$ C. A. Perroni and A. Liebsch, Phys. Rev. B 74, 134430 (2006).

${ }^{30}$ F. Hansteen, A. Kimel, A. Kirilyuk, and T. Rasing, Phys. Rev. Lett. 95, 047402 (2005).

${ }^{31}$ A. Kirilyuk, A. V. Kimel, T. Rasing, Rev. Mod. Phys. 82, 2731 (2010).

${ }^{32}$ H. Luttgemeir, H. G. Bohn, and M. Brajczewska, J. Magn. Magn. Mater. 21, 289 (1980).

${ }^{33}$ G. V. Kozlov, S. P. Lebedev, A. A. Mukhin, A. S. Prokhorov, I. V. Fedorov, A. M. Balbashov, and I. Yu. Parseglov, IEEE Trans. Magn. 29, 3443 (1993).

${ }^{34}$ K. N. Kocharyan, R. M. Martirosyan, V. G. Prpryan, and E. L. Sarkisyan, Sov. Phys. JETP 59, 373 (1984).

${ }^{35}$ W. J. Tabor and F. S. Chen, J. Appl. Phys. 40, 2760 (1969).

${ }^{36}$ N. Ray and U. V. Waghmare, Phys. Rev. B 77, 134112 (2008).

${ }^{37}$ H. Němec, F. Kadlec, P. Kužel, L. Duvillaret, and J. L. Coutaz, Opt. Comm. 260, 175 (2006). 